\title{
GEOMETRY INDUCED LOCALIZATION OF ELECTRONS IN QUANTUM PILLARS
}

\author{
IOANNIS G. TIGELIS ${ }^{a, *}$ and JOHN P. XANTHAKIS ${ }^{b}$ \\ ${ }^{a}$ Physics Department, University of Athens, Panepistimiopolis, Zografou, Athens, \\ 15784 Greece; ${ }^{\mathrm{b}}$ Electrical and Computer Engineering Department, National \\ Technical University of Athens, Polytechnioupolis, Zografou, Athens, \\ 15773 Greece
}

(Received 10 May 1996; In final form 17 June 1996)

\begin{abstract}
We investigate the energies and wavefunctions of a structure consisting of one small cubic box centered on top of another larger with a uniform potential inside. This model is a representation of a quantum pillar on a substrate, a nanostructure with potential device applications. We find that the eigenstates are localized in one or the other of the boxes. Those in the large box are very well localized while those in the small are moderately so. Their energy and localization are studied with respect to the ratio of sides of the two boxes and compared with the results obtained with the small box closed by all sides.
\end{abstract}

\section{INTRODUCTION}

Quantum Pillars, i.e., arrays of nanometer boxes deposited on a substrate of the same material as the pillars, have been produced in the laboratory using both Si and GaAs [1], [2]. Their size ranges from $500 \AA$ (width) $\times$ $800 \AA$ (height) in GaAs to $50 \AA$ (width) $\times 1 \mu \mathrm{m}$ (height) in Si [2]. All of them have interesting optical properties with potential device applications [1], [3]. The Si pillars, in particular, exhibit visible luminescence at 620 $\mathrm{nm}$, which is very similar to the one observed in porous $\mathrm{Si}$, thus enhancing the view that this phenomenon is a quantum size effect and is not related to some surface defect or impurity complex [3], [4].

\footnotetext{
*Corresponding author.
} 
A theoretical question that needs to be answered before Effective Mass Theory (EMT) or even a supercell calculation can be applied to this $\mathrm{Si}$ system [5], is whether the pillars can be dissociated from the substrate, given that the bottom of the pillars is in contact with a "sink" of Bloch states, the substrate. To put it in simpler terms, given that the particles in the nanometer boxes are localized in " $21 / 2$ " dimensions (D), can one be sure that they are localized in 3D. A similar but more complex problem arises with the structure of porous Si [3].

The purpose of this paper is to answer this question. We will examine both the degree of localization inside the boxes and the movement of the corresponding energy levels with respect to the size of the boxes. Finally, tentative comparisons will be made with the predictions of EMT for a pillar confined at both ends.

\section{THEORY}

It should be obvious from the discussion of the previous section that the determining factor in the modulation of the electronic structure of quantum pillars is the smallness of the size involved. Therefore, as a first attempt, we may put the potential everywhere constant-since the pillar and the substrate are of the same material-and use the free electron model to simulate the conduction (or valence) band of the system. Orthogonal symmetry is used for convenience. Furthermore, since the pillars are quite separated from one another, we consider only one pillar centered on top of its corresponding portion of substrate. This structure is shown in Figure 1.

The general prescription for solving such problems is well known. One expands in terms of the particular solutions in each subspace-the pillar and the substrate - and by matching the solution at the interface one obtains an algebraic equation that gives the eigenvalues and then the eigenvectors.

Shroedinger's equation with the potential equated to zero reads

$$
\frac{\hbar^{2}}{2 \mathrm{~m}_{0}} \nabla^{2} \Psi t=-\mathrm{E} \Psi \text { or } \nabla{ }^{2} \Psi=-\mathrm{K}^{2} \Psi
$$

where $m_{0}$ is the electron mass. 


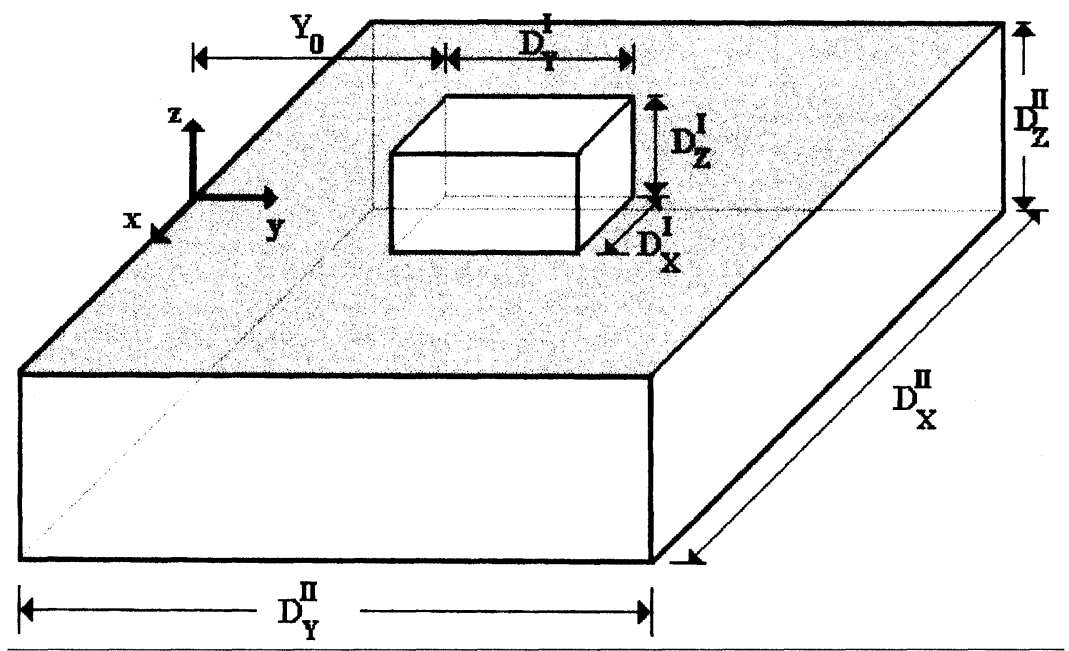

FIGURE 1 Schematic view of a pillar on a substrate.

In cartesian coordinates, this is separable and therefore any particular solution of (1) must be of a product form. It is not very difficult to deduce that the general solution, which has zero value at the outer boundaries, is (see Fig. 1 for notation)

$$
\Psi(\mathrm{x}, \mathrm{y}, \mathrm{z})=\sum_{\mathrm{m}=1}^{\infty} \sum_{\mathrm{n}=1}^{\infty} \mathrm{A}_{\mathrm{mn}} \sin \left[\mathrm{K}_{\mathrm{x}}^{\mathrm{I}}\left(\mathrm{x}+\frac{\mathrm{D}_{\mathrm{x}}^{\mathrm{I}}}{2}\right)\right] \sin \left[\mathrm{K}_{\mathrm{y}}^{\mathrm{I}}\left(\mathrm{y}-\mathrm{y}_{0}\right)\right] \Theta_{1}(\mathrm{z})
$$

where

$$
\Theta_{1}(\mathrm{z})= \begin{cases}\sin \left[\mathrm{K}_{\mathrm{z}}^{\mathrm{I}}\left(\mathrm{z}-\mathrm{D}_{\mathrm{z}}^{\mathrm{I}}\right)\right] & \text { if }\left(\mathrm{K}_{\mathrm{z}}^{\mathrm{I}}\right)^{2}>0 \\ \sinh \left[\mathrm{t}_{\mathrm{z}}^{\mathrm{I}}\left(\mathrm{z}-\mathrm{D}_{\mathrm{z}}^{\mathrm{I}}\right)\right] & \text { if }\left(\mathrm{K}_{\mathrm{z}}^{\mathrm{I}}\right)^{2}=-\left(\mathrm{t}_{\mathrm{z}}^{\mathrm{I}}\right)^{2}<0\end{cases}
$$

and

$$
\mathrm{K}_{\mathrm{x}}^{\mathrm{I}}=\mathrm{m} \pi / \mathrm{D}_{\mathrm{x}}^{\mathrm{I}}, \mathrm{K}_{\mathrm{y}}^{\mathrm{I}}=\mathrm{n} \pi / \mathrm{D}_{\mathrm{y}}^{\mathrm{I}} \text { and }\left(\mathrm{K}_{\mathrm{z}}^{\mathrm{I}}\right)^{2}=\frac{2 \mathrm{~m}_{0} \mathrm{E}}{\hbar^{2}}-\left(\mathrm{K}_{\mathrm{x}}^{\mathrm{I}}\right)^{2}-\left(\mathrm{K}_{\mathrm{y}}^{\mathrm{I}}\right)^{2}
$$


inside the upper box I $(\mathrm{z}>0)$ with a similar expression holding inside the lower box II $(\mathrm{z}<0)$. Note that the coefficients in the expression for $\Psi$ in the lower box will be denoted by $B_{i j}(i=1,2, \ldots, \infty$ and $j=1,2, \ldots, \infty)$.

By requiring that $\Psi$ and $\partial \Psi / \partial \mathrm{z}$ are continuous at the interface, one obtains two equations for the $\mathrm{A}_{\mathrm{mn}}$ and $\mathrm{B}_{\mathrm{ij}}$ :

$$
B_{i j}=\frac{4 \Theta_{1}(0)}{\Theta_{2}(0) D_{x}^{I I} D_{y}^{I I}} \sum_{m=1}^{\infty} \sum_{n=1}^{\infty} A_{m n} I_{i m}^{x} I_{j n}^{y}
$$

and

$$
A_{m n}=\frac{4 \dot{\Theta}_{2}(0)}{\dot{\Theta}_{1}(0) D_{x}^{I} D_{y}^{I}} \sum_{i=1}^{\infty} \sum_{j=1}^{\infty} B_{i j} I_{i m}^{x} I_{j n}^{y}
$$

where

$$
\begin{gathered}
\Theta_{2}(z)= \begin{cases}\sin \left[K_{z}^{\mathrm{II}}\left(z+D_{z}^{\mathrm{II}}\right)\right] & \text { if }\left(\mathrm{K}_{\mathrm{z}}^{\mathrm{II}}\right)^{2}>0 \\
\sinh \left[\mathrm{t}_{\mathrm{z}}^{\mathrm{II}}\left(\mathrm{z}+\mathrm{D}_{\mathrm{z}}^{\mathrm{II}}\right)\right] & \text { if }\left(\mathrm{K}_{\mathrm{z}}^{\mathrm{II}}\right)^{2}=-\left(\mathrm{t}_{\mathrm{z}}^{\mathrm{II}}\right)^{2}<0\end{cases} \\
\mathrm{I}_{\mathrm{im}}^{\mathrm{X}}=\int_{-\mathrm{D}_{\mathrm{x}}^{\mathrm{i}} / 2}^{\mathrm{D}_{\mathrm{x}}^{\mathrm{I}} / 2} \sin \left[\mathrm{K}_{\mathrm{i}}^{\mathrm{II}}\left(\mathrm{x}+\frac{\mathrm{D}_{\mathrm{x}}^{\mathrm{II}}}{2}\right)\right] \sin \left[\mathrm{K}_{\mathrm{m}}^{\mathrm{I}}\left(\mathrm{x}+\frac{\mathrm{D}_{\mathrm{x}}^{\mathrm{I}}}{2}\right)\right] \mathrm{dx} \\
\mathrm{I}_{\mathrm{j} \mathrm{y}}^{\mathrm{y}}=\int_{\mathrm{y}_{0}}^{\mathrm{y}_{0}+\mathrm{D}_{\mathrm{y}}^{\mathrm{I}}} \sin \left(\mathrm{K}_{\mathrm{j}}^{\mathrm{II}} \mathrm{y}\right) \sin \left[\mathrm{K}_{\mathrm{n}}^{\mathrm{I}}\left(\mathrm{y}-\mathrm{y}_{0}\right)\right] \mathrm{dy}
\end{gathered}
$$

Note that $\Theta_{i}(z)(i=1,2)$ denotes the derivative function with respect to z, i.e., $\Theta_{i}(z)=\partial \Theta_{i} / \partial z$.

A single equation relating only the $A_{m n}$ can now be obtained, whose determinant, which is a function of $\mathrm{K}$, must be zero. Hence, the eigenvalues are obtained (cf eq. 4) and then the expansion coefficients $A_{m n}$ and $B_{i j}$ are determined. Note that the above expressions are exact within the freeelectron model. 


\section{RESULTS AND DISCUSSION}

The distribution in energy and $\mathrm{K}$ content of the 10 lowest states for a cubic pillar $\left(\mathrm{D}_{x}^{\mathrm{I}}=\mathrm{D}_{y}^{\mathrm{I}}=\mathrm{D}_{z}^{\mathrm{I}}=\mathrm{D}^{\mathrm{I}}\right)$ and substrate system $\left(\mathrm{D}_{x}^{\mathrm{II}}=\mathrm{D}_{y}^{\mathrm{II}}=\right.$ $\left.\mathrm{D}_{z}^{\mathrm{II}}=\mathrm{D}^{\mathrm{II}}\right)$ with ratio of sizes $1: 2$ is shown in Table I. The first column is the energy, the second is the degeneracy of the state, and the third is the ratio of the most significant $A_{m n}$ (denoted by $A$ ) to the most significant $B_{i}$ (B), the square of which gives a measure of the localization of the wavefunction in each box. This is so because in the majority of eigenstates examined, there were only one $A_{m n}$ and one $B_{i j}$ dominant, the other coefficients being an order of magnitude or more smaller. Judging by the ratio $(\mathrm{A} / \mathrm{B})^{2}$ we may say that the majority of these states are very well localized in the lower box, while the ones lying in the upper one are only weakly localized. Note that the energies of the latter lie scattered inside the spectrum of states of the lower box, as expected, and are not separated from them.

The lowest state inside the pillar is of particular physical significance. We expect it to move up in energy and be more localized as the pillar becomes smaller. This is indeed shown in the following two figures. In Figure 2, we plot the degree of localization $(\mathrm{A} / \mathrm{B})^{2}$ of this state as a function of $x=D^{I I} / D^{I}$. Note that it does not depend on the absolute values of $\mathrm{D}^{\mathrm{I}}$ and $\mathrm{D}^{\mathrm{II}}$, but only on their ratio, cf. eq. (5)-(6). It can be seen that the

TABLE I Energy levels (first column), their degeneracy (second column) and ratio of most significant $A_{m n}$ to most significant $B_{i j}$ for two cubic boxes with sides $5 a_{0}$ and $10 a_{0}$. energies are in Ryd. For longer sides the energies must be scaled by $\left(a_{0} / D^{I I}\right)^{2}$

\begin{tabular}{lcc}
\hline Energy $($ Ryd $)$ & \multicolumn{2}{c}{$D^{I}=5 a_{0}, D^{I I}=10 a_{0}, y_{0}=2.5 a_{0}$} \\
\cline { 2 - 3 } & Degeneracy & $A_{m m} / B_{i j}$ \\
\hline 0.286 & 1 & $\mathrm{~A}_{11} / \mathrm{B}_{11}=0.023$ \\
0.545 & 1 & $\mathrm{~A}_{11} / \mathrm{B}_{11}=0.17$ \\
0.589 & 2 & $\mathrm{~A}_{12} / \mathrm{B}_{12}=0.001$ \\
0.874 & $\mathrm{~A}_{12} / \mathrm{B}_{12}=0.004$ \\
0.881 & 2 & $\mathrm{~A}_{11} / \mathrm{B}_{11}=2.47$ \\
0.887 & 1 & $\mathrm{~A}_{11} / \mathrm{B}_{11}=0.0001$ \\
1.084 & 1 & $\mathrm{~A}_{11} / \mathrm{B}_{13}=0.152, \mathrm{~B}_{11} / \mathrm{B}_{13}=0.944$ \\
1.084 & 1 & $\mathrm{~A}_{13} / \mathrm{B}_{13}=0.0$ \\
1.125 & 1 & $\mathrm{~A}_{11} / \mathrm{B}_{11}=2.78$ \\
1.187 & 1 & $\mathrm{~A}_{22} / \mathrm{B}_{22}=0.0004$ \\
\hline
\end{tabular}




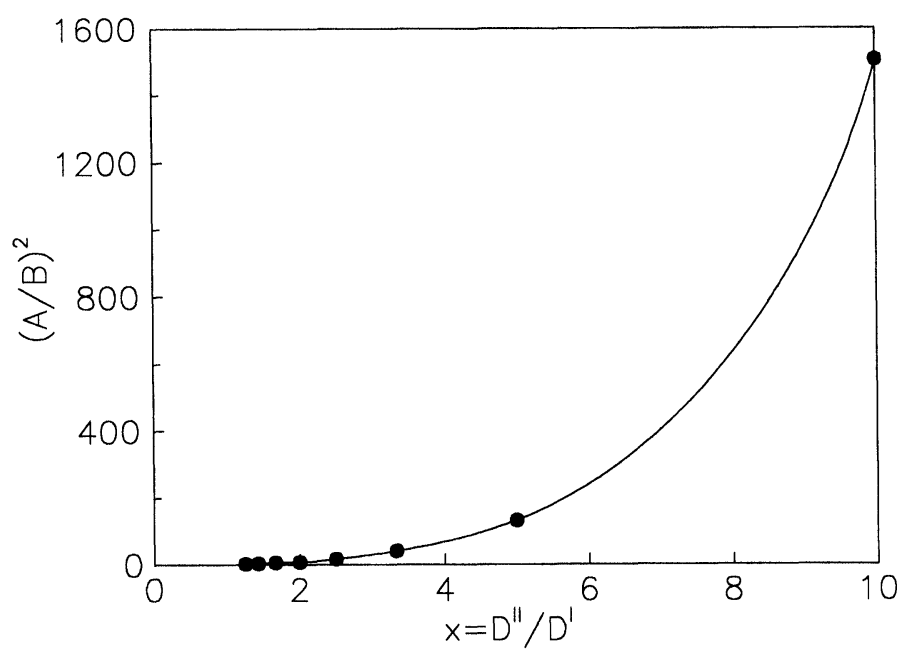

FIGURE 2 Variation of the localization factor $(A / B)^{2}$ w.r.t $x=D^{I I} / D^{I}$.

degree of localization increases monotonically with $\mathrm{x}$ and takes large values at small $\mathrm{x}$. It is hard to see that this trend will not continue at much higher $\mathrm{x}$.

In Figure 3, we plot the energy of the same state as a function of $x$ together with the value that it would have if the upper box were bound by all sides. It can be seen that the two curves do not seem to converge, our values being consistently lower than those of the "particle in the box" problem or EMT for that matter. Although the percentage difference fluctuates around $20 \%$, it represents a very small absolute value for $\mathrm{x} \gg 10$, see the units of Table I.

Additional information is given in Figure 4, where we compare the charge density $|\Psi|^{2}$ of the lowest state localized in each box along the axis of symmetry ( $\mathrm{z}$ direction) of the combined structure for two different values of $x$, namely $x=10 / 3$ and $x=10 / 1$. We observe that, whereas the localization of the ground state in the lower box (substrate) is almost complete (Figures $4 \mathrm{a}$ and $4 \mathrm{~b}$ ), considerable spilling over into the lower box occurs in the charge density of the "ground" state of the upper box (pillar) (Figures $4 \mathrm{c}$ and $4 \mathrm{~d}$ ). As the size of the pillar is reduced this charge 


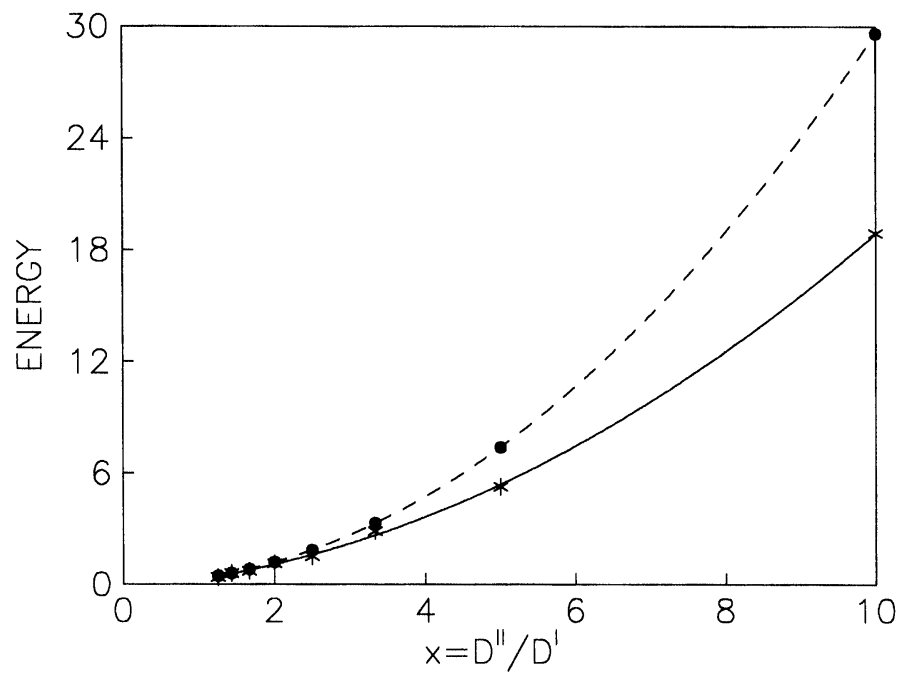

FIGURE 3 Variation of the energy of the lowest level localized in the smaller box w.r.t $\mathrm{x}$ as calculated by our method (solid curve) and assuming that the box is closed by all sides (broken curve). Units are as in Table I.

density is gradually being emptied from the substrate, see the low z part of Figure $4 \mathrm{~d}$. On the other hand even at very high $\mathrm{x},|\Psi|^{2}$ is never zero at the interface.

The above analysis allows us to draw a simple picture of electrons inside quantum pillars. The electrons are indeed localized inside the pillars provided one can think of the latter as effectively extending by a certain amount into the substrate. This amount diminishes as the pillar becomes smaller compared to the substrate and will be negligible in the experiments performed so far. Note that this result is independent of the absolute size of either the pillar or the substrate and depends solely on their ratio. Furthermore, the charge density is not evenly distributed but electrons prefer the region near the interface. The fact that the effective size of each pillar is considerably greater (for small ratio of sizes) than the geometric one may help explain why our calculated values of the energy of this state do not approach those of a particle in a box and are consistently lower. 

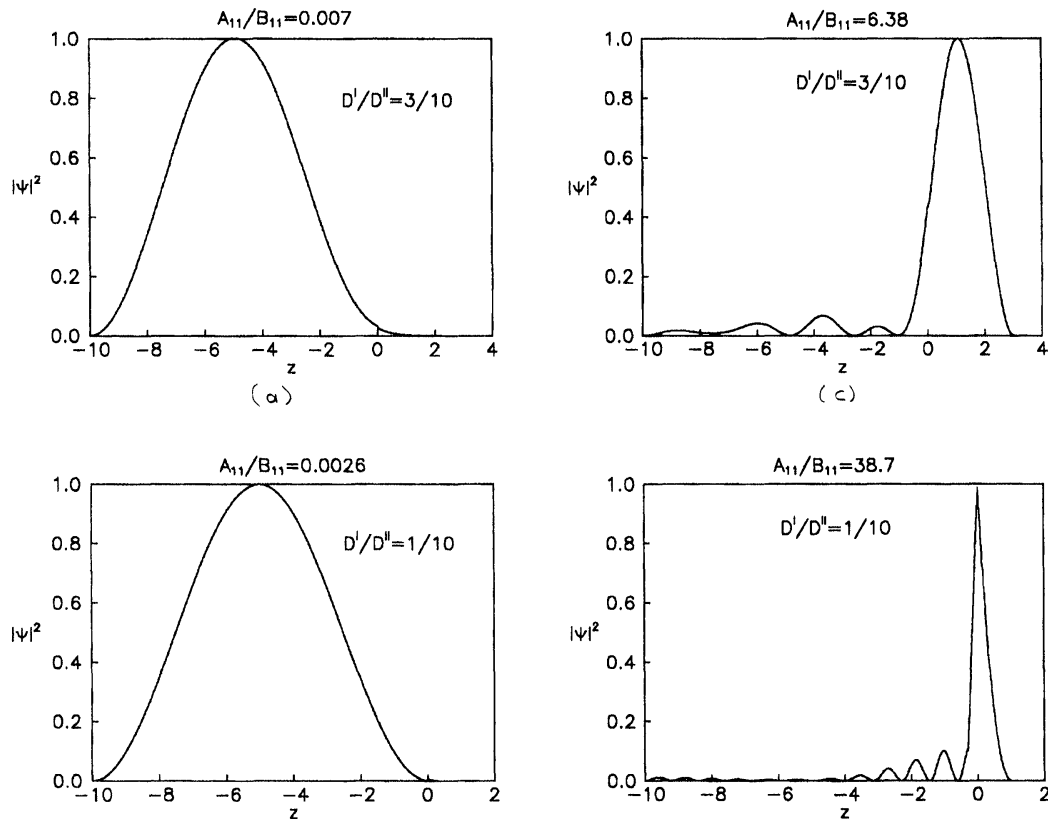

(b)

(d)

FIGURE 4 Charge density $|\Psi|^{2}$ along the axis of symmetry of the two boxes:

a: lowest state localized in larger box, ratio of sides $3: 10$

b: lowest state localized in larger box, ratio of sides $1: 10$

c: lowest state localized in smaller box, ratio of sides $3: 10$

d: lowest state localized in smaller box, ratio of sides $1: 10$.

\section{References}

[1] M. Green, M. Garcia-Parajo, F. Khaleque and R. Murray, Quantum pillar structures on $n^{+}$GaAs fabricated using "natural" lithography, Appl. Phys. Lett. 62, pp. 264, 1993.

[2] A. G. Nassiopoulos, S. Grigoropoulos, E. Gogolides and D. Papadimitriou, Visible luminescence from one- and two-dimensional silicon structures produced by conventional lithographic and reactive ion etching techniques, Appl. Phys. Lett. 66, pp. 1115, 1995.

[3] A. G. Cullis and L. T. Canham, Visible light emission due to quantum size effects in highly porous crystalline silicon, Nature (London) 353, pp. 335, 1991.

[4] L. T. Canham, Luminescent bands and their proposed origins in highly porous silicon, Phys. Stat. Sol. B 190, pp. 9, 1994.

[5] A. J. Read, R. J. Needs, K. J. Nash, L. T. Canham, P. D. J. Calcott and A. Qteish, First-principles calculations of the electronic properties of silicon quantum wires, Phys. Rev. Lett. 69, pp. 1232, 1992. 

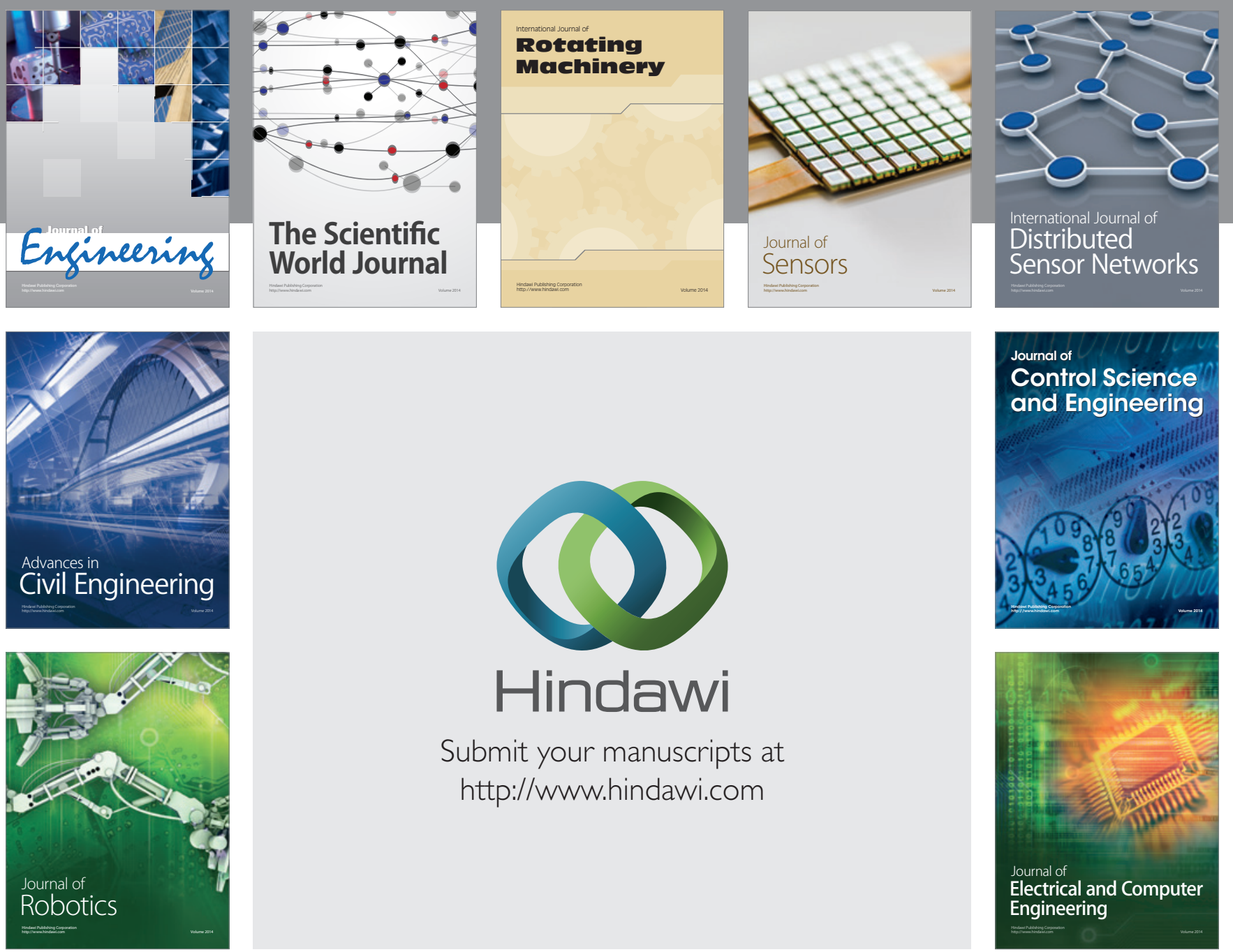

Submit your manuscripts at

http://www.hindawi.com
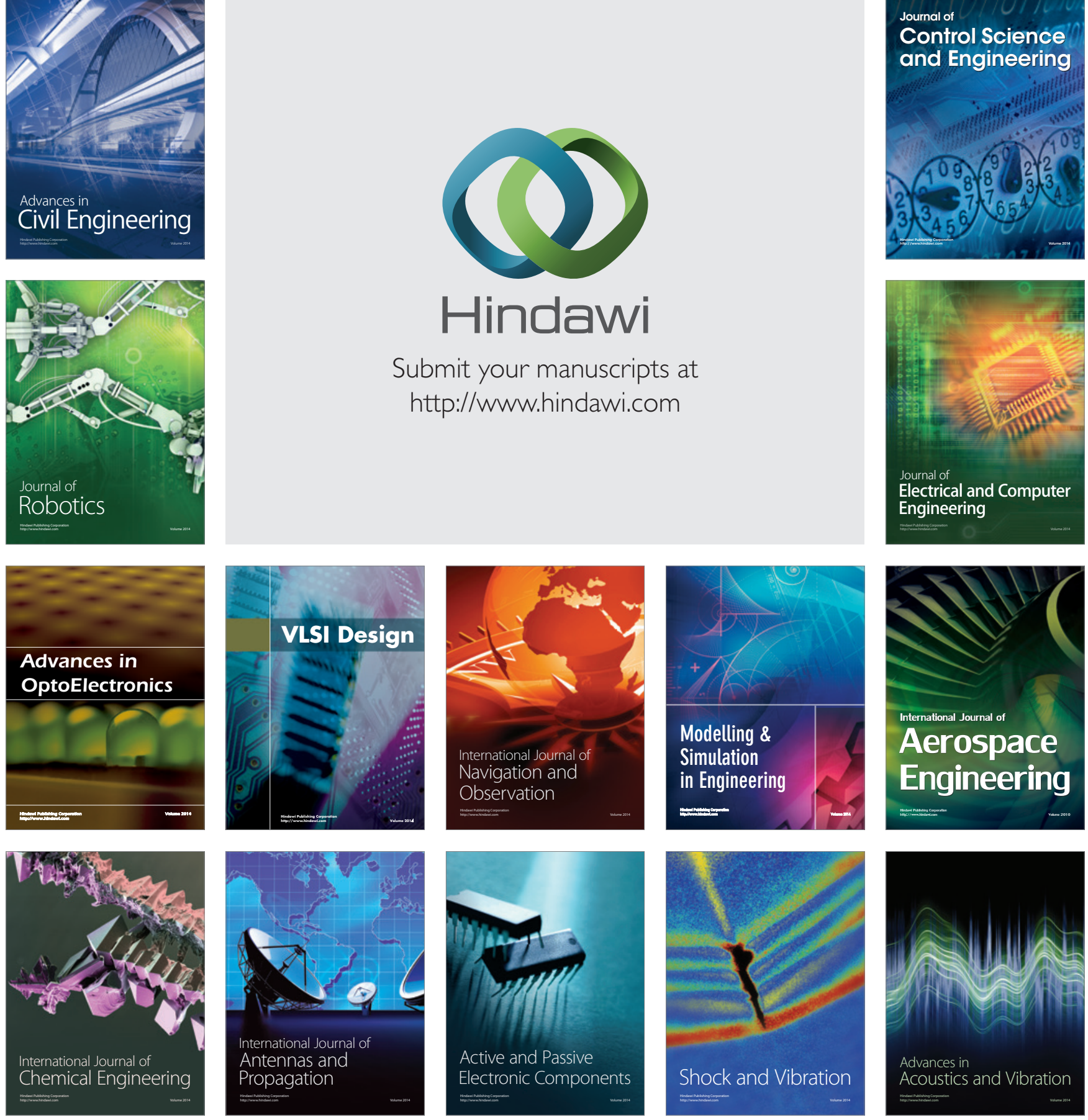